\title{
Motherhood in Transition - Who Cares? Eine qualitative Analyse medialer Anerkennungsstrukturen von Mutterschaft und Care-Arbeit
}

\author{
Natalie Berner
}

Keywords: Mutterschaft, Care-Arbeit, Diskursanalyse, Spätmoderne Valorisierungsstrukturen, Multimodalität, Leitmedien, TV-Werbung, Instagram

\section{Abstract}

Strukturelle Veränderung im Sinne einer Care-Revolution muss Bestandteil jedes transformativen Nachdenkens sein. Dieser Beitrag fragt, welche Anerkennungsstrukturen von Mutterschaft und Care-Arbeit medial wirksam sind. Hierfür wurden exemplarische Analyseeinheiten aufverschiedenen medialen Diskursebenen qualitativ untersucht (Leitmedien, TV-Werbung und Instagram im Zeitraum von 2016 bis 2018). Die punktuelle Analyse des Mediendiskurses offenbart 1) eine Lücke in Bezug auf die sprechenden Personen, 2) eine nach wie vor normativ aufgeladene Mutterfigur, die medial eingesetzt wird und als Sehnsuchtsobjekt dient, 3) die Ästhetisierung, Kommodifizierung und Vereinheitlichung von Mutterschaft und 4) die Erschöpfung und Frustration, die symptomatisch für Care-Geber*innen zu sein scheint.

Natalie Berner: Motherhood in Transition - Who Cares? Eine qualitative Analyse medialer Anerkennungsstrukturen von Mutterschaft und Care-Arbeit. In: Nils S. Borchers, Selma Güney, Uwe Krüger und Kerem Schamberger (Hrsg.): Transformation der Medien - Medien der Transformation. Verhandlungen des Netzwerks Kritische Kommunikationswissenschaft. Frankfurt am Main: Westend 2021. DOI: https://doi.org/10.53291/VUAT3055.

Natalie Berner | Ludwig-Maximilians-Universität München I natalie. berner@ifkw.Imu.de 


\section{Einführung}

Die »Große Transformation «, die der Wissenschaftliche Beirat der Bundesregierung 2011 veranschlagt hatte, setzt sich für eine klima- und sozial gerechte, ökologisch wirtschaftende Weltgesellschaft ein. Allerdings werden die Transformationsidee und der Begriff $»$ Nachhaltigkeit« überproportional häufig mit dem Themenkomplex um Klima und Umwelt assoziiert. Der Care-Bereich hingegen, also die Fürsorge, Hinwendung und Pflege des Lebendigen, steht weniger stark im Fokus, obwohl hier ebenfalls ganz unmittelbar die Existenzgrundlagen des menschlichen Seins betroffen sind. Während der Raubbau an der Natur mit medientauglichen Bildern transportiert werden kann, die gerodete Wälder, verschmutzte Ozeane und schmelzende Pole zeigen, vollzieht sich der Raubbau am Menschen unbemerkt und in komplexer Chiffrierung. Übersetzt in sozialwissenschaftliches Vokabular steht er hier für strukturelle Bedingungen, die soziale Ungleichheit, (Selbst-)Ausbeutung und die Unsichtbarmachung großer Teile geleisteter Care-Arbeit fördern.

Im Gegensatz dazu (oder als Reaktion) fordern Barbara Thiessen und Paula-Irene Villa schon lange die strukturelle Anerkennung der Angewiesenheit des Menschen (auf Menschen) als Conditio humana (Thiessen und Villa 2010). Dabei plädieren sie unter anderem dafür, das Recht, Sorge zu geben und versorgt zu werden, im Sozialgesetzbuch als Grundrecht zu verankern. Das Konzept der »Care-Revolution« (Winker 2015) legt in diesem Sinne Maßstäbe für eine solidarische Gesellschaft an und ist zu verstehen als »[...] eine politische Transformationsstrategie, die anknüpfend an die Erkenntnisse feministischer Politik die grundlegende Bedeutung der Sorgearbeit ins Zentrum stellt und darauf abzielt, das gesellschaftliche Zusammenleben ausgehend von menschlichen Bedürfnissen zu gestalten. Damit wird Care-Arbeit, die in den meisten politischen Strategien ebenso wie in den ökonomischen Theorien keine Rolle spielt, als Bezugspunkt der Gesellschaftsveränderung gewählt.« (ebd., 144)

Der Beitrag blickt vor diesem Hintergrund diskursanalytisch auf massenmedial vermittelte Deutungs- und Anerkennungsstrukturen, die unter den Bedingungen der Medienlogik (Karidi 2017) produziert werden. Anerkennungsstrukturen sind hier analog zu Reckwitz (2019) als »Valorisierungsstrukturen « zu verstehen, die sich in hochgradig dynamischen, gesellschaftlichen Prozessen herausbilden und »Elementen der Welt« (ebd., 27) Wert zuschreiben beziehungsweise absprechen. 
Dieser konfliktbeladene »Prozess des ^doing value« (ebd.) manifestiert sich auch in medialen Anerkennungsstrukturen. Welchen Lebensformen, Alltagsvorstellungen oder Praktiken wird Wert zugesprochen beziehungsweise aberkannt? Welcher "Stil des Lebens « (ebd., 55) ist medial sichtbar oder gerade unsichtbar und somit wertlos? Gesellschaftliche Anerkennungsstrukturen können sich auf Freizeitgestaltung, persönliche Beziehungen, die Geschlechterordnung, das Familienleben, die politische Orientierung und prinzipiell auf alle Formen des Seins beziehen (ebd.).

Gegenstand dieser Betrachtung ist das Thema Mutterschaft und die damit verbundene öffentliche Bewertung von Care-Arbeit. Die Ergebnisse basieren auf einer qualitativen Diskursanalyse, die kategoriengeleitet aufgesetzt und ausgewertet wurde (Löblich 2016; Meyen 2019). Das Untersuchungsmaterial setzt sich aus 282 Analyseeinheiten zusammen, die drei unterschiedlichen medialen Diskursebenen zugehören: 55 entstammen Leitmedien, 27 der TV-Werbung und 200 der digitalen Plattform Instagram. Diese Heterogenität ermöglicht die Herausarbeitung kanalübergreifender und somit symptomatischer Regelmäßigkeiten. Theoretische Grundlagen sind dabei zum einen die diskursanalytischen Prämissen nach Foucault (1981) kombiniert mit der gesellschaftsdiagnostischen Analyse der Spätmoderne nach Reckwitz (2017; 2019) und der wissenschaftlichen Care-Debatte beziehungsweise der Idee einer Care-Revolution (Winker 2015). Die Ergebnisse beschränken sich im Rahmen dieses Beitrags auf punktuelle Skizzierungen und sind als Auskopplung eines größer angelegten Dissertationsprojekts zu sehen.

\section{Problemaufriss: Mutterschaft und Care in der Spätmoderne}

\subsection{Spätmoderne Valorisierungsstrukturen und ihre Kehrseiten}

Nach Reckwitz (2019) leidet die Spätmoderne an einem systematischen Ungleichgewicht. Sie ist geprägt durch die Gleichzeitigkeit von sozialem Auf- und Abstieg sowie kultureller Auf- und Abwertung (ebd., 16). Das heißt: Den Gewinner*innen des spätmodernen Bewertungssystems stehen Verlierer*innen gegenüber und die Aufwertung 
einer bestimmten Lebensweise kommt nicht ohne die Entwertung einer anderen aus. Reckwitz bezeichnet dies als Doppelstruktur der Spätmoderne, als zwei Seiten einer Medaille, die nicht voneinander zu trennen sind. Zwar handelt es sich laut ihm bei der Entwertung um nichtintendierte Handlungsfolgen, die weder bewusst herbeigeführt noch explizit gewünscht werden - aber dennoch in aller Konsequenz existieren. So sind etwa Singularitätsprozesse, die das Individuum mit allerlei Freiheiten und vermeintlichem Entfaltungsspielräumen ausstatten, implizit auch eine Abwertung all dessen, was »standardisiert und massenhaft im Hintergrund verschwindet« (Reckwitz 2019, 17). Viele gesellschaftliche Spannungen lassen sich über die Doppelstruktur von Valorisierung erklären: einseitige oder fehlende Anerkennung und Negierung der Kehrseiten von bessergestellten sozialen Positionen.

Die Kehrseiten spätmoderner Valorisierungsstrukturen zeigen sich nach Reckwitz vielerorts: ihm zufolge wertet der global vernetzte kognitiv-kulturelle Kapitalismus komplexe Güter mit hoher Innovationsdynamik und Kreativität zum Vorteil der Hochqualifizierten auf (ebd., 16), während gleichzeitig einfache Dienstleistungen, Routinejobs und repetitive Arbeiten von Niedrigqualifizierten mit geringem Prestige und sozialer Sicherheit ihren Wert verlieren (ebd.). Die Kehrseite der Akademisierung und des Profilierungswettbewerbs von Schulen, Universitäten und Absolventen um Exzellenz und Alleinstellung zeige sich außerdem in der Abwertung von niedrigen und mittleren Bildungsabschlüssen (ebd., 18). In der digitalen Welt wiederum erhielten eine geringe Zahl von Individuen Aufmerksamkeit und Wertschätzung im Übermaß (ebd.). Diesen Gewinner*innen stellt Reckwitz all jene gegenüber, »die unsichtbar bleiben, die schlecht vernetzt oder isoliert sind, denen es an Anerkennung mangelt - oder die gar ins Zentrum negativer Aufmerksamkeit wie Verachtung oder Hass geraten « (ebd.). Gleichzeitig sei das Individuum selbst erschöpft vom eigenen »Lebensmodell der erfolgreichen Selbstverwirklichung «, das die Gestaltung von »Alltag, Biographie, Freizeit und Familie zu einer ambitionierten Herausforderung « (ebd.) mache und ein hohes Enttäuschungs- und Frustrationspotenzial berge. $\mathrm{Ob}$ das Leben als gelungen empfunden wird, ist nach Reckwitz von fragilen Maßstäben abhängig: dem subjektiven Erleben und der eigenen psychischen Befriedigung (ebd., 21) - Finde ich das gut? Macht mir das Spaß? Dieses Modell werde allerdings nur von einer neuen Mittelklasse, dem »Leitmilieu der Spätmoderne« (ebd., 17), getragen. Die Kehrseite findet sich ihm zufolge 
in der »subtilen kulturellen Abwertung oder sozialen Deklassierung, welche die traditionelle Mittelklasse und prekäre Klasse erfahren« (ebd., 18).

\subsection{Mediale Valorisierungsstrukturen und ihre Bedeutsamkeit}

Dieses Reckwitz'sche »Bewertungssystem der Spätmoderne « findet in Form von besagten Valorisierungsstrukturen ${ }^{1}$ seine Entsprechung im medialen Diskurs. ${ }^{2}$ Die Medienöffentlichkeit schafft Realität, indem sie bewertet. Sie positioniert und hierarchisiert das Subjekt und schafft Vergleichsräume (Arendt und Brosius 2019). Valorisierungsstrukturen werden über Mediendiskurse institutionalisiert und entfalten ihre Wirkung als Orientierungssysteme, die den Mitgliedern einer Gesellschaft bekannt sind. Sie prägen den Wissensvorrat, formen Mythen sowie das kulturelle Gedächtnis und stecken Handlungsspielräume ab (Berger und Luckmann 2016). Valorisierungsstrukturen bieten der Kommunikationswissenschaft eine vielversprechende Analysekategorie, da sie eine elegante Brücke zwischen soziologischer Gesellschafts- und Gegenwartsdiagnose sowie kritischer Medieninhaltsforschung bilden. Sie sind zudem durch die jeweilige Kanallogik geformt und somit in ihren Bedeutungspotenzialen eingeschränkt. Wie im medialen Diskurs Wert zugesprochen beziehungsweise verweigert werden kann, ist den medialen Produktionsbedingungen und den Regeln der Medienlogik unterworfen. Hier stellt sich die Frage, welche Folgen es für das Subjekt hat, wenn sich gesamtgesellschaftlich wirksame Anerkennungsstrukturen in dem Korsett medialer Produktionslogiken formieren. Umso dringlicher wird sie, je weiter fortgeschritten die »reflexive Modernisierung « (Beck et al. 2014) ist, je weniger traditionsgeleitet und vorgeschrieben die Handlungsspielräume des Einzelnen sind und je mehr mediale Valorisierungsstrukturen diese Lücken in den subjektiven Orientierungssystemen füllen können.

1 Kontroverse um Anerkennung oder Umverteilung vgl. Fraser und Honneth (2003).

2 Diskursbegriff nach Foucault (1981). 


\subsection{Care-Arbeit und Mutterschaft als spätmoderner »Aggressionspunkt«}

Mutterschaft und Mutterschaftspraxen stehen nun in besonderem Maße im Zentrum der spätmodernen (Neu-)Formierung entlang der Kategorien »Gender « und »Care (Thiessen 2019, 1146). Die Klammer um das »Neu« ist an dieser Stelle wichtig, weil die Geschichte zeigt, dass die Auslegung von Mutterschaft schon immer von Wandel durchzogen war. Brüche und Neuinterpretationen von Mutterschaft, Elternschaft und Familie begleiten diese Konzepte schon seit ihrer Prägung (Thurer 1995; Thiessen 2019; 2020; Schütze 1986; Vinken 2011). Daher lohnt ein diskursanalytischer Blick, der bestehende Herrschaftsverhältnisse als Grundlage dominanter Deutungen in den Fokus nimmt und sich ihrer Kontingenz bewusst ist. Nachdem sich in Deutschland im Zuge der Industrialisierung und der Herausbildung der bürgerlichen Kleinfamilie eine stark geschlechtshierarchische Arbeitsteilung etabliert hatte, nahmen die Ansprüche und die normative Aufgeladenheit von gelungener Mutterschaft im Zeitverlauf stetig zu (Schütze 1986; Thiessen und Villa 2010). Die voranschreitende Ökonomisierung sowie der steigende Stellenwert des Kindes innerhalb der Familie und die neuentdeckte Lebensphase »Kindheit« führten zu einer fortsetzenden Professionalisierung der Mutterrolle (Thiessen 2020). Gleichzeitig wird das traditionelle Ernährermodell abgelöst von einer Ordnung, welche die Erwerbstätigkeit aller erwachsenen Menschen vorsieht. Das »Adult-Worker-Modell« als erklärtes Ziel familienpolitischer Maßnahmen (Winker 2015) steht für eine normative Verschiebung in eine Richtung, in der alle Menschen zu »geschlechtslosen Marktteilnehmern« (Soiland 2019, 102) reformiert werden, »die dem Markt unabhängig von ihren reproduktiven Fürsorgeaufgaben zur Verfügung stehen sollen« (ebd.).

Zugleich erfahren Care-Tätigkeiten, die meist unbezahlt und informell von Frauen geleistet wurden und werden, als Form von Arbeit wenig gesellschaftliche Anerkennung. Entsprechend gering fällt auch die Entlohnung für staatliche oder privatwirtschaftliche personennahe Tätigkeiten in Betreuung und Pflege aus (Winker 2015, 23). Während der Bedarf an Care-Arbeit steigt (unter anderem durch den demographischen Wandel und eine wachsende Zahl an Alleinstehenden), nehmen die Möglichkeiten für Sorge und Pflege ab - beispielsweise aufgrund von weniger Mehrgenerationenhaushalten, der Flexibilisierung der Arbeitswelt, steigender Mobilität und fehlenden Geschwistern (Eh- 
lert 2016). Care-Praktiken folgen zusätzlich einer Eigenlogik, die sie für Rationalisierungsmechanismen sperrig machen. Sie sind zeitintensiv und lassen sich nicht standardisieren oder verkürzen, ohne an Qualität zu verlieren (Winker 2015). Menschlicher Zeiteinsatz und oftmals physische Präsenz machen Care-Tätigkeiten makroökonomisch teuer. Der Ressourceneinsatz kann nicht unbedingt durch Innovation effektiver oder günstiger gestaltet werden. So gerät Care zu einem »Aggressionspunkt« (Rosa 2018) des spätmodernen Menschen, da diese Tätigkeiten nicht widerstandsfrei »verfügbar« sind beziehungsweise sich nicht in die Maxime einer entgrenzten Lebensweise integrieren lassen. Gelöst wird der hohe Ressourcenaufwand durch billige Arbeitskräfte aus dem Ausland, die oft in prekären Verhältnissen angestellt sind und eigene Familien zurücklassen (»care-chain«; Lutz und Palenga-Mollenbeck 2012). Außerdem lagert man Care-Tätigkeiten oftmals aus (Kinderbetreuung, haushaltsnahe Dienstleistungen, Pflegedienste, digitale Dienste), was zu intersektionalen Schieflagen führt, da sich die Möglichkeiten von Familien und Care-Empfänger*innen entlang von Ressourcen, Milieus oder Regionen unterscheiden (Thiessen 2020; Winker 2015). Eine lohn- und statuswirksame Aufwertung von Care-Arbeit ist daher unerlässlich, um prekäre Auswege aus der Care-Krise zu beheben (Soiland 2019). Vor diesem Hintergrund stellt sich die Frage, wie Valorisierungsstrukturen um Mutterschaft und Care-Arbeit im Mediendiskurs beschaffen sind, welche Machtverhältnisse sich dabei zeigen und wie die mediale Produktionslogik Deutungsstrukturen formt. Eine „Care-Revolution«, die sich an menschlichen Bedürfnissen und insbesondere an der Sorge füreinander orientiert, kann als zentrale »Transformationsstrategie (Winker 2015) nur über entsprechende mediale Valorisierungsstrukturen getragen und vorangebracht werden.

\section{Forschungsleitende Fragen kompakt}

Welche Valorisierungsstrukturen zeigen sich im Untersuchungsmaterial in Bezug auf Mutterschaft und Care-Arbeit?

- Was sagt das über geltende Herrschaftsverhältnisse aus? Wo sind Lücken und Leerstellen?

- Welchen Einfluss hat die Kanallogik? 


\section{Untersuchungsanlage}

Für die qualitative Analyse des Mediendiskurses wurden im Rahmen der Grobanalyse 282 Diskursfragmente berücksichtigt (Tab. 1). Diese entstammen unterschiedlichen Medienkanälen (Leitmedien, TV-Werbung und Instagram als digitale Plattform), um den Diskurs möglichst breit einzufangen. Alle Fragmente wurden im Zeitraum zwischen 2016 und 2018 veröffentlicht. Die Auswahl der Printartikel erfolgte über eine Schlagwortsuche (Mutter, Mutterschaft, Eltern, Elternschaft, Familie, Geburt) und anschließende Gewichtung nach den Kriterien Zentralität und Relevanz. Die Instagram-Posts wurden über die Anzahl der Follower*innen und die Selbstbeschreibung, in der Mutterschaft vorkommen musste, selektiert. Die Auswahl der Werbespots erfolgte über TVMonitoring von Oktober 2018 bis Januar 2019. Hierfür wurden diese gezielt in den reichweitenstärksten Fernsehkanälen gesichtet und die Erhebung durch eine Recherche in Werbedatenbanken ergänzt. Das Selektionskriterium bei den Werbespots bestand ebenfalls darin, dass eine beliebige Deutung von Mutterschaft auffindbar sein musste.

\section{1 Überblick über die Materialbasis}

Tab. 1: Die Materialbasis im Überblick.

\begin{tabular}{|l|l|l|}
\hline Leitmedien & 55 Artikel & $\begin{array}{l}\text { Süddeutsche Zeitung (SZ), Frankfurter Allgemeine Zeitung } \\
\text { (FAZ), Die Tageszeitung (taz), Die Welt, Die Zeit, Der Spiegel }\end{array}$ \\
\hline TV-Werbung & 27 Werbespots & $\begin{array}{l}\text { Nahrungsmittel, Pharmazie, Handel, Reinigungsmittel, } \\
\text { Babyprodukte }\end{array}$ \\
\hline Instagram & 200 Posts & $\begin{array}{l}\text { Sprecher*innen, die sich aufgrund der Selbstbeschreibung } \\
\text { in die Kategorien »Celebrity Moms«, »Working Moms « oder } \\
\text { »Momfluencer « einteilen lassen }\end{array}$ \\
\hline
\end{tabular}

Für die Auswertung wurde ein Kategoriensystem verwendet (Tab. 2). Die Auswertung erfolgte nach Jäger und Jäger (2007) in zwei Stufen: In der Grobanalyse wurden grundsätzliche Regelmäßigkeiten festgestellt und typische Fälle bestimmt. Diese gingen in die Feinanalyse ein und wurden daraufhin in Text- und Bildebene auf die latenten und manifesten Bedeutungsstrukturen untersucht. 


\subsection{Kategoriensystem}

Tab. 2: Das Kategoriensystem, Grundlage für die empirische Untersuchung.

\begin{tabular}{|l|l|}
\hline Kanallogik & \multicolumn{2}{|l|}{} \\
\hline Diskursposition & $\begin{array}{l}\text { Sprecher*innenhintergrund } \\
\text { Problembezüge/Gegenstandskonzeptualisierung } \\
\text { Anerkennungsstrukturen (Valorisierung) }\end{array}$ \\
\hline Strategien & $\begin{array}{l}\text { Sprachliche Mittel, ideologisches Framing } \\
\text { Kollektivsymbolik, Bildkomposition }\end{array}$ \\
\hline
\end{tabular}

Die Kanallogik berücksichtigt dabei die unterschiedlichen Kontexte, in denen das Diskursfragment entsteht. So folgt ein Printartikel in der SZ einer anderen Logik als ein Instagram-Post oder eine TV-Werbung. Dies hängt mit den Produktionsbedingungen, den Möglichkeiten der Vermittlung (Text, Bild, audiovisuell) sowie den Nutzungskontexten der Medieninhalte zusammen. In der Kategorie Diskursposition werden einerseits die strukturellen Vorbedingungen der Sprecher*innen erhoben sowie die inhaltliche Konzeptualisierung und Bewertung des Gegenstands »Mutter«. Welche visuellen und sprachlichen Mittel dabei zur Anwendung kommen, erfasst die Kategorie Strategien. Die theoretische Fundierung des hier verkürzt dargestellten Kategoriensystems geht zurück auf die Formationsregeln von Foucault (1981) sowie die anwendungsorientierte Adaption von Jäger und Jäger für die Sprachund Sozialforschung (2007). 


\section{Ergebnisse}

\subsection{Sprecher*innenschaft und Leerstellen}

In den hier untersuchten Diskursfragmenten zeichnet sich die Sprecher*innenschaft durch Homogenität hinsichtlich der Ressourcenausstattung und sozialen Position aus. So kann für die untersuchten Analyseeinheiten im erhobenen Zeitraum festgehalten werden: Deutungshoheit hat die neue Mittelklasse gemäß Reckwitz' Definition. So richten sich die Problembezüge, vor denen Mutterschaft medial diskutiert wird, an der Lebenswelt dieser homogenen Gruppe aus. Folglich sind die Interessenlagen und Relevanzsysteme anderer Teile der Bevölkerung medial weniger bis gar nicht sichtbar. Es werden Leerstellen im Mediendiskurs in Bezug auf Diskurszugang bestimmter Sprecher*innenpositionen augenscheinlich. Welche Problembezüge beim Thema Mutterschaft und Care-Arbeit bei der alten Mittelkasse oder der prekären Klasse vorherrschen, findet wenig bis gar keine Beachtung. Den Verlierer*innen des Strukturwandels, die durch eine kulturelle Defensive oder strukturell unsichere Lebensbedingungen geprägt sind, steht als »Kehrseite « keine mediale Entsprechung im vorliegenden Untersuchungsmaterial gegenüber. Das Perfide daran ist aber, dass es oft gerade der unsichtbare Ressourceneinsatz dieser Personen ist, auf dem der spätmoderne Lebenswandel auf der »Gewinner*innenseite« aufbaut. Der Modus der Gleichzeitigkeit und der Synchronität divergierender Rollenansprüche gelingt langfristig nur durch »Ressourcenzukauf«. Haushaltshilfen, Erzieher*innen und Großeltern, die Care-Tätigkeiten übernehmen und dadurch \#WorkingMom ermöglichen, erhalten dafür keine äquivalente Valorisierung. Diese konzentriert sich auf das Subjekt, das im Sinne des spätmodernen Bewertungssystems reüssiert. Hier zeichnen sich Asymmetrien im medialen Bewertungssystem ab, welches ein enges Verständnis von Erfolg und Leistung zur Vergleichskategorie macht, dabei die Frage nach Vorbedingungen und Ressourcen vernachlässigt und so soziale Ungleichheit fördert. 


\subsection{Anerkennungsstrukturen zu Mutterschaft und Care-Arbeit}

Vergleicht man die Konzeptualisierung von Mutterschaft über die Kanäle hinweg, zeigt sich deutlich die Wirksamkeit sich widersprechender Anerkennungsstrukturen. Zum einen erfolgt Anerkennung nach den Kriterien der spätmodernen Steigerungslogik. Das heißt, Mutterschaft wird dann positiv valorisiert, wenn ein Streben nach Selbstverwirklichung, Statuserhalt und sozialem Erfolg parallel zur Sorgearbeit hinzutritt. Gleichzeitig wird Mutterschaft vor allem visuell nach archetypischen Mustern, also mit starken genderspezifischen Markern von Weiblichkeit sowie einer symbiotischen Darstellung von Mutter und Kind (vgl. Abb. 1), inszeniert und instrumentalisiert.
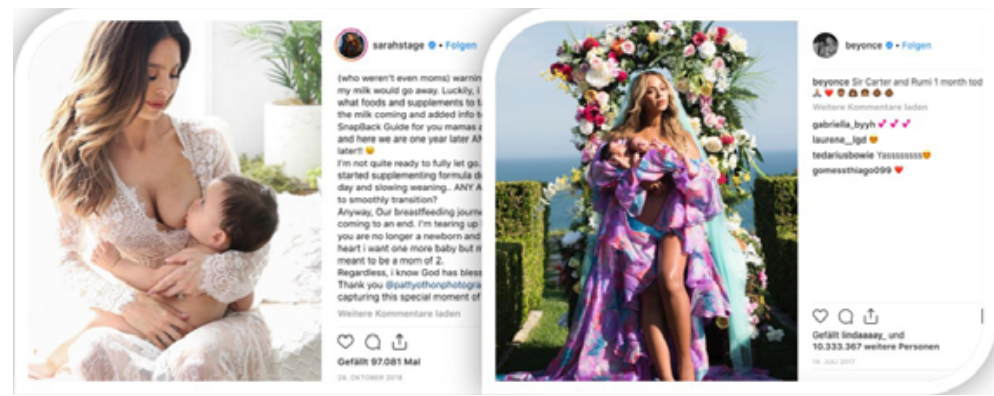

Abb. 1: Beispiele Instagram: Sarah Stage (28.10.2018), Knowles

(14.07.2017).

Das Konzept »Mutterschaft « gerät auf visueller Ebene zur Personifizierung von Fürsorge-Tätigkeiten (Nahrung, Pflege, Aufmerksamkeit, liebevolle Zuwendung) und ist damit stark mit Care-Arbeit verwoben. Nur durch Präsenz, Verfügbarkeit sowie die Bezogenheit auf das Kind scheint die Bedeutung von Mutterschaft visuell transportierbar zu sein. Das steht gerade im diametralen Widerspruch zur Ressourcenverhandlung, die vor allem in den Leitmedien geführt wird und in denen die Bemühung zu beobachten ist, Definitionsmachtverhältnisse (Beck 2017) hinsichtlich Mutterschaft und Mutterschaftspraxen neu zu justieren. Denn in den Analyseeinheiten aus den Leitmedien wird genau diese visuell dominant transportierte Bindung von Ressourcen aufgebrochen und zum Gegenstand der diskursiven Umsortierung. Die Ressource »Mutter« als sich sorgende, sich kümmernde steht zur 
Disposition - und wird gleichermaßen verteidigt wie angegriffen. Dies geschieht sowohl mit Blick auf biopolitische Fragen: Wer darf und soll biologisch oder sozial Mutter sein (etwa in Bezug auf Leihmutterschaft, Reproduktionsmedizin, Rechte von gleichgeschlechtlichen Paaren, Ressourcenausstattung), aber vor allem auch in Bezug auf Verteilungsfragen: Welcher Ressourceneinsatz und welche Emotionen sind in Mutterschaftspraxen legitim? Wie müssen diese in Partner- oder Elternschaft verteilt sein?

Darüber hinaus werden legitime staatliche Maßnahmen diskutiert, die den Ressourceneinsatz einerseits unter Paaren und andererseits zwischen unbezahlter Care-Arbeit und Lohnarbeit ausgleichen sollen. Mutterschaft ist in den Leitmedien folglich auf drei unterschiedlich intimen Ebenen von Diskurskämpfen betroffen: 1) Wer darf beziehungsweise soll Mutter sein? 2) Welcher zeitliche und emotionale Ressourceneinsatz ist für Mutterschaftspraxen gesellschaftlich erwünscht? 3) Durch welche ökonomischen und politischen Steuerungsmaßnahmen lässt sich der Ressourceneinsatz in Fürsorgearbeit mit der Verfügbarkeit für den Arbeitsmarkt zusammenbringen? Eine Doppelstruktur in der diskursiven Herstellung von Mutterschaft (Bewertung des Ressourceneinsatzes nach spätmoderner Marktlogik versus archetypische Care-Ikone) zeigt sich auch innerhalb der einzelnen Diskursfragmente. Hervorzuheben ist dabei der Umstand, dass Care-Arbeit genderspezifisch unterschiedlich valorisiert wird. Beispielsweise bewertet die Materialbasis Väter positiv, die Fürsorge vor beruflichen Aufstieg stellen, während dies bei Frauen nicht der Fall ist (Clauß 2016). Die Wahrnehmung von Sorgetätigkeiten durch eine Frau wird in den Analyseeinheiten nur in Kombination mit anderen Errungenschaften positiv valorisiert. Care-Arbeit ist in der Bewertungshierarchie der Spätmoderne anderen Bezugssystemen nachgeordnet: Das Sich-Sorgen um Kinder oder andere Care-Empfänger ist weniger wert als der Ressourceneinsatz Zeit auf anderen Märkten.

Als Folge der unterschiedlichen Valorisierung von Care- und Lohnarbeit stellt sich in der medialen Inszenierung von Mutterschaft ein Modus der Gleichzeitigkeit und Synchronität ein. Diesem steht ein Erschöpfungsdiskurs gegenüber, welcher die Kehrseite subjektiver Selbstoptimierungsversuche zeigt und als Ventil einer erschöpften neuen Mittelklasse dient, die an den eigenen Maßstäben leidet, ohne diese strukturell in Frage zu stellen oder ins Verhältnis zu setzen.

Gleichzeitigkeit und Erschöpfung sind diskursdynamisch als Reaktion zu bewerten, die den dominanten, aufgeblähten und einseitigen 
spätmodernen Anerkennungskriterien gegenüberstehen. So hat Petra Schmidt (2014) die moderne Mutter bereits als »Pionierin der Spätmoderne«, als »Avantgarde der Subjektivierung von Arbeit« beschrieben, welche »selbstkontrolliert, selbstrationalisiert, selbstökonomisiert « all das mit subjektiven und externalisierten Ressourcen aufzufangen versucht, was an steigenden gesellschaftlichen Erwartungen an das Individuum herangetragen wird.
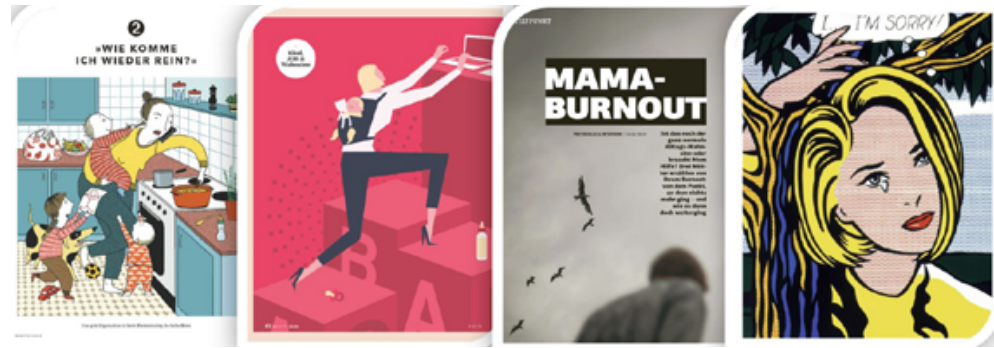

Abb. 2: Beispiele aus Brigitte (2018), Brigitte Mom (Sims 2018, Minner 2018) und Der Spiegel (Voigt 2016).

\subsection{Einfluss der Kanallogik auf den medialen Diskursebenen}

Auf verschiedenen medialen Diskursebenen kommt es dabei zu unterschiedlichen Formen der Ausprägung dieser Doppelstruktur. Es wird im Folgenden beispielhaft auf die Kanallogik von Instagram und TV-Werbung eingegangen.

Die Werbung positioniert die Mutter gemäß Kanallogik strategisch vorteilhaft für das beworbene Produkt. Dabei wird ihr in erster Linie die Funktion zugesprochen, sich um alle Belange des zwischenmenschlichen Um- und Versorgens zu kümmern. Sie ist primär auf das Wohlergehen der anderen (Kind und Partner) bedacht und kümmert sich, wenn überhaupt, zuletzt um sich selbst. Während Kind und Partner Fürsorge empfangen, wird die Mutter stets als Gebende, Wissende, Sorgende, Nährende konzeptualisiert. Dies geht einher mit einem stark vereindeutigten Frauenbild, das ebenfalls keinerlei Irritationen zulässt und die Mutter nur dann explizit positiv valorisiert, wenn sie ein attraktives Erscheinungsbild aufweist, einen kreativen und humorvollen Umgang mit den Unwägbarkeiten des Alltags und der Erziehung findet 
sowie »gute Entscheidungen für die Versorgung der Familie und des Kindes trifft. Überlappende Rollenansprüche, Ressourcenknappheit und Erschöpfung werden nur dann dargestellt, wenn das beworbene Produkt eine Lösung für die divergierenden Ansprüche bietet, wie etwa Nahrungsergänzungsmittel und Mittel zur psychischen oder physischen Leistungssteigerung. Es zeigen sich im Bereich der TV-Werbung so Diskursfragmente im Sinne der spätmodernen Steigerungslogik bei gleichzeitiger Verfestigung traditioneller Rollenbilder. Einerseits wird eine archetypische Form von Mutterschaft hergestellt, die sich ausschließlich mit Care-Tätigkeiten beschäftigt und so Sehnsüchte nach Versorgung und Geborgenheit befriedigt. Andererseits wird auf den Erschöpfungsdiskurs Bezug genommen, um eine kommerzielle Lösung anzubieten. Durch die Reaktivierung der Leistungsfähigkeit kann das Subjekt den spätmodernen Ansprüchen wieder gerecht werden, so das Versprechen.

Auf Instagram zeigt sich die Doppelstruktur auf andere Weise. Zunächst ist die Logik von Instagram geprägt durch Ästhetisierung und Kommodifizierung - also das Zur-Ware-Machen - von Mutterschaft (Schmidt 2015). Digitale Plattformen wie Instagram begrenzen durch ihre Eigenlogik den Vorstellungsraum des Möglichen und tragen zu einer Vereinheitlichung der Deutungsstrukturen bei. So werden alle Modi von Mutterschaft in das Korsett der Ästhetisierung und des visuell Darstellbaren eingefügt, während das Unästhetische oder visuell nicht Vermittelbare von vorneherein ausgeschlossen sind. Diskursfragmente auf Instagram sind immer zugleich kreative Akte, denen eine bestimmte Wirkungsabsicht vorausgeht. So zeigen sich Leerstellen bei der Darstellung von Normalität und Durchschnittlichkeit, aber auch in Bezug auf die »abgewandte« Mutter (genervt, aggressiv, abweisend, wütend). Insbesondere die »Momfluencer« monetarisieren erfolgreich die Inszenierung der eigenen sozialen Rolle und fokussieren sich hier gänzlich auf eine care-basierte Darstellung von Mutterschaft. Bemerkenswert ist der hohe Einfluss des Kindes auf die Bildkomposition. Ist das Kind abgebildet, scheint nur eine zugewandte, irritationsfreie Form von Mutterschaft darstellbar zu sein. Widersprüche und Inkonsistenzen zeigen sich allein auf der textlichen Ebene, deren Aussagen oft konträr zur Bildlogik stehen.

Die implizite Bedeutungsstruktur der attraktiven Mutter und Frau bleibt hochgradig aktiv. Werden Themen wie Depression, Ermüdung und mentale Überfrachtung angesprochen, ist das Kind nicht auf dem Bild zu sehen. Stattdessen bleibt die Mutter auf sich selbst zurückge- 
worfen. Auch auf Instagram wird Mutterschaft im Modus der Gleichzeitigkeit hergestellt: Die Mutter zeigt sich in der Sphäre der Erwerbstätigkeit, während nur der Text auf die Mutterschaft Bezug nimmt (und umgekehrt). So wird polychrones Zeitmanagement und Entgrenzung auf Instagram zum normalisierten Modus von Mutterschaft, ohne dass die Ressourcen sichtbar werden, die es hierfür benötigt. Divergierende Zeitrhythmen von Arbeits- und Familienwelt lassen sich scheinbar widerspruchsfrei integrieren (Schmidt 2014).

\subsection{Fazit und transformativer Ausblick}

Der Mediendiskurs spielt eine entscheidende Rolle, wenn es um die nachhaltige Transformation gesellschaftlicher Strukturen im Sinne caregerechter Lebens- und Arbeitsbedingungen geht. Die punktuelle Analyse des Mediendiskurses offenbart 1) eine Lücke in Bezug auf die sprechenden Personen, 2) eine nach wie vor wirksame, normativ aufgeladene Mutterfigur, die medial eingesetzt wird und als Sehnsuchtsobjekt dient (Mutter als Versorgende, Liebende), 3) die Ästhetisierung und Kommodifizierung und Vereinheitlichung von Mutterschaft und 4) die Erschöpfung und Frustration, die symptomatisch für Care-Geber*innen zu sein scheint. Ein kritischer Blick auf strukturelle Zusammenhänge bleibt überwiegend aus. Es werden ungelöste Konflikte hinsichtlich der Anforderungen und Eigenschaften von Fürsorgearbeit sowie der Verschränkung von Mutterschaft mit Marktimperativen deutlich. Die Medienlogik fördert die Individualisierung von Konflikten (Erschöpfungsdiskurs) auf der einen und die Kommodifizierung und Ästhetisierung von Mutterschaft auf der anderen Seite. Diskurspositionen, die für Machbarkeit und »Autonomie-Fetischismus « (Thiessen und Villa 2010) stehen, untergraben die gesellschaftliche Aufwertung von fürsorgebasierten Beziehungen, die sich jenseits von Marktimperativen bewegen. Doch nur durch eine solche Aufwertung können sich intersektional gerechte Strukturen ausbilden.

Die Problemlagen der Großen Transformation müssen im Zusammenhang wahrgenommen und bearbeitet werden. Ebenso verhält es sich mit Care-Arbeit und Mutterschaft. Um diesen spätmodernen Aggressionspunkt aufzulösen, bedarf es neuer gesellschaftlicher Zielgrößen wie »Zeitwohlstand « (Habermann et al. 2015). Es gilt, Strukturen zu formen, welche die Möglichkeit, Sorge zu geben und zu empfangen, in den Mittelpunkt stellen - unabhängig von Alter, Gender, Ethnie oder 
sozialem Status. Denn das Menschliche nimmt hier - in der Angewiesenheit - seinen Anfang und sein Ende. Die Möglichkeit, in Verbundenheit zu leben und zu sterben - auch daran muss sich die Große Transformation messen lassen dürfen.

\section{Literatur}

Arendt, Florian, und Hans-Bernd Brosius. 2019. Soziale Vergleiche und Medienwandel. MedienJournal. DOI: https://doi.org/10.24989/medienjournal. v43i2.1732. Zugegriffen: 17. Juli 2020.

Beck, Ulrich. 2017. Die Metamorphose der Welt. Berlin: Suhrkamp.

Beck, Ulrich, Anthony Giddens, und Scott Lash (Hrsg.). 2014. Reflexive Modernisierung: eine Kontroverse. 6. Auflage. Frankfurt am Main: Suhrkamp.

Berger, Peter L., und Thomas Luckmann. 2016. Die gesellschaftliche Konstruktion der Wirklichkeit: eine Theorie der Wissenssoziologie. 26. Auflage. Frankfurt am Main: Fischer Taschenbuch.

Clauß, Anna. 2016. Sie ist wieder da. Der Spiegel Nr. 11, 44-49.

Ehlert, Nancy. 2016. Familienpolitik als Carepolitik für Ältere. https://www.bpb. $\mathrm{de} /$ politik/innenpolitik/familienpolitik/218864/carepolitik-fuer-aeltere? $\mathrm{p}=$ all . Zugegriffen: 17. Juli 2020.

Fraser, Nancy, und Axel Honneth. 2003. Umverteilung oder Anerkennung? Eine politisch-philosophische Kontroverse. Frankfurt am Main: Suhrkamp.

Foucault, Michel. 1981. Archäologie des Wissens. Frankfurt am Main: Suhrkamp.

Habermann, Friederike, Hartmut Rosa, Frigga Haug, Niko Paech, Lena Kirschenmann, und Felix Wittmann. 2015. Zeitwohlstand: Wie wir anders arbeiten, nachhaltig wirtschaften und besser leben. 2. Auflage. München: Oekom.

Jäger, Margret, und Siegfried Jäger. 2007. Deutungskämpfe: Theorie und Praxis kritischer Diskursanalyse. Wiesbaden: VS Verlag für Sozialwissenschaften.

Karidi, Maria. 2017. Medienlogik im Wandel: die deutsche Berichterstattung 1984 und 2014 im Vergleich. Wiesbaden: Springer VS.

Knowles, Beyoncé. 2017. Posts [@beyonce] vom 14. Juli. Instagram-Profil. https:// www.instagram.com/beyonce/?hl=de. Zugegriffen: 15. Mai 2020.

Löblich, Maria. 2016. Theoriegeleitete Forschung in der Kommunikationswissenschaft. In: Handbuch nicht standardisierte Methoden in der Kommunikationswissenschaft, herausgegeben von Stefanie Averbeck-Lietz und Michael Meyen, 6779. Wiesbaden: Springer VS.

Lutz, Helma, und Ewa Palenga-Mollenbeck. 2012. Care Workers, Care Drain, and Care Chains: Reflections on Care, Migration, and Citizenship. Social Politics: International Studies in Gender, State \& Society. DOI: https://doi.org/10.1093/sp/ jxr026.

Meyen, Michael, Maria Löblich, Senta Pfaff-Rüdiger, und Claudia Riesmeyer. 2019. Qualitative Forschung in der Kommunikationswissenschaft. Eine praxisorientierte Einführung. Wiesbaden: Springer VS. 
Minner, Claudia. 2018. Mama-Burnout. Brigitte Mom Nr. 1, 116-122.

Reckwitz, Andreas. 2019. Das Ende der Illusionen: Politik, Ökonomie und Kultur in der Spätmoderne. Berlin: Suhrkamp.

Rosa, Hartmut. 2018. Mehr Welt! Umrisse einer Kritik der Verfügbarkeit. Wien, Salzburg: Residenz.

Sims, Gill. 2018. Raus aus dem Chaos. Brigitte Mom Nr. 3, 82-86.

Schmidt, Petra. 2014. Total Quality Mama: Mutterschaft aus der Perspektive Arbeit. München: Utz.

Schütze, Yvonne. 1986. Die gute Mutter: zur Geschichte des normativen Musters »Mutterliebe«. Theorie und Praxis der Frauenforschung, Band 3. Bielefeld: B. Kleine.

Soiland, Tove. 2019. Ökonomisierung - Privatisierung: die verdeckte Unterseite neoliberaler Restrukturierungen und ihre Implikationen für die Geschlechterforschung. In: Handbuch Interdisziplinäre Geschlechterforschung, herausgegeben von Beate Kortendiek, Birgit Riegraf und Katja Sabisch, 95-104. Wiesbaden: Springer VS. DOI: https://doi.org/10.1007/978-3-658-12496-0_13.

Stage, Sarah. 2018. Posts [@sarahstage] vom 28. Oktober. Instagram-Profil. https://www.instagram.com/sarahstage/?hl=de. Zugegriffen: 15. Mai 2020.

Thiessen, Barbara. 2019. Mutterschaft: Zwischen (Re-)Naturalisierung und Diskursivierung von Gender und Care. In: Handbuch Interdisziplinäre Geschlechterforschung, herausgegeben von Beate Kortendiek, Birgit Riegraf und Katja Sabisch, 1141-1149. DOI: https://doi.org/10.1007/978-3-658-12496-0_64.

Thiessen, Barbara. 2020. Doing Family while Doing Care: Wie Familie gemacht wird. Gendergraphien XI: Geschlecht und Familie. Vortrag vom 7. Februar. LMU München.

Thiessen, Barbara, und Paula-Irene Villa. 2010. Entweder - oder? Mutterschaft zwischen Fundamentalismen und vielschichtigen Praxen. querelles-net. DOI: https://doi.org/10.14766/875.

Thurer, Shari. 1995. Mythos Mutterschaft: Wie der Zeitgeist das Bild der guten Mutter immer wieder neu erfindet. München: Droemer Knaur.

Vinken, Barbara. 2011. Die deutsche Mutter: Der lange Schatten eines Mythos. Frankfurt am Main: S. Fischer.

Voigt, Claudia. 2016. Gut, besser, mittelmäßig. Der Spiegel Nr. 6, 130-132.

»Wie komme ich wieder rein?«. 2018. Brigitte Nr. 4, 92.

Winker, Gabriele. 2015. Care Revolution: Schritte in eine solidarische Gesellschaft. $X$-Texte. Bielefeld: Transcript.

\section{Open Access}

Dieser Beitrag erscheint unter der Creative-Commons-Lizenz CC BY-ND 3.0 DE: https://creativecommons.org/licenses/by-nd/3.0/de/. 